EUROPHYSICS LETTERS

15 May 1999

Europhys. Lett., 46 (4), pp. 425-430 (1999)

\title{
Cumulant expansion for systems with large spins
}

\author{
K. KLAdKo ${ }^{1}$ (甘) P. Fulde ${ }^{2}$, and D. A. GARAnin ${ }^{2}$ **) \\ 1 Condensed Matter Physics Group and Center for Nonlinear Studies, \\ Theoretical Division, Los Alamos National Laboratory, \\ MS-B258, Los Alamos, New Mexico 87545, USA \\ 2 Max-Planck-Institut für Physik komplexer Systeme, \\ Nöthnitzer Str. 38, D-01187 Dresden, Germany
}

(received 23 November 1998; accepted 12 March 1999)

PACS. 05.30.-d - Quantum statistical mechanics.

PACS. 75.10.Jm- Quantized spin models.

PACS. 75.10.Hk - Classical spin models.

\begin{abstract}
A method is proposed for obtaining a systematic expansion of thermodynamic functions of spin systems with large spin $S$ in powers of $1 / S$. It uses the cumulant technique and a coherent-state representation of the partition function $\mathcal{Z}$. The expansion of $\mathcal{Z}$ in terms of cumulants yields an effective classical Hamiltonian with temperature-dependent quantum corrections. For the Heisenberg quantum Hamiltonian, they have a non-Heisenberg form. The effective Hamiltonian can be solved by methods familiar for classical systems.
\end{abstract}

In recent years considerable interest in condensed matter physics has been focusing on the properties of new magnetic materials, such as magnetic molecules with a large effective spin $S$, or intermetallic compounds containing magnetic layers or chains. For the latter, most of the theoretical studies deal with $S=1 / 2$ systems which, in the one-dimensional case, allow for analytical solutions using special methods such as the Bethe Ansatz or Jordan-Wigner fermion representation of spins. On the other hand, constructing expansions in powers of $1 / S$ for systems with large values of $\mathrm{S}$, which are not constrained to special lattice geometries or to low temperatures, has not received sufficient attention.

The aim of this paper is to show that quantum corrections to the classical thermodynamics of spin systems with large or moderate $S$ can be calculated to an arbitrary order in the quasiclassical parameter $1 / S$. This is achieved by using a cumulant expansion technique and working in the basis of spin coherent states. The results which can be obtained by our method are valid for temperatures down to the quantum energy scale $\tilde{J} / S$, where $\tilde{J} \equiv J S^{2}$ is the classical energy scale and $J$ is the exchange interaction. They are complementary to those following from the spin-wave theory (SWT) based either on the Holstein-Primakoff or path-integral formalisms, which works in the range $T \lesssim \tilde{J}$ (see, for example, ref. [1]).

Averages or matrix elements $\langle\ldots\rangle$ can be expressed through cumulants $\langle\ldots\rangle^{c}$ as follows

$$
\begin{aligned}
& \langle A\rangle=\langle A\rangle^{c}, \quad\left\langle A_{1} A_{2}\right\rangle=\left\langle A_{1} A_{2}\right\rangle^{c}+\left\langle A_{1}\right\rangle\left\langle A_{2}\right\rangle, \\
& \left\langle A_{1} A_{2} A_{3}\right\rangle=\left\langle A_{1} A_{2} A_{3}\right\rangle^{c}+\left\langle A_{1}\right\rangle\left\langle A_{2} A_{3}\right\rangle^{c}+\left\langle A_{2}\right\rangle\left\langle A_{1} A_{3}\right\rangle^{c}+\left\langle A_{3}\right\rangle\left\langle A_{1} A_{2}\right\rangle^{c}+\left\langle A_{1}\right\rangle\left\langle A_{2}\right\rangle\left\langle A_{3}\right\rangle,
\end{aligned}
$$

$\left(^{*}\right.$ ) cnls.lanl.gov/ kladko/; kladko@lanl.gov

$\left({ }^{* *}\right)$ www.mpipks-dresden.mpg.de/ garanin/; garanin@mpipks-dresden.mpg.de 
etc., where $A_{i}$ are classical stochastic variables or quantum-mechanical operators. The averaging above is performed over a classical distribition function or weighed over quantum states. A detailed discussion of cumulants, with an emphasis on quantum systems, can be found in refs. [2, 3]. Cumulants can be obtained by differentiation of a generating function, i.e., from

$$
\left\langle A_{1} \ldots A_{N}\right\rangle^{c}=\left.\frac{\partial}{\partial \lambda_{1}} \cdots \frac{\partial}{\partial \lambda_{N}} \ln \left\langle e^{\lambda_{1} A_{1}} \ldots e^{\lambda_{N} A_{N}}\right\rangle\right|_{\lambda_{1}=\ldots=\lambda_{N}=0}
$$

in contrast to averages $\langle\ldots\rangle$, which are given by a similar expression without logarithm. By setting $A_{i}=A$, multiplying by $\lambda^{n} / n$ !, and summing over $n=1, \ldots, \infty$ one obtains the identity

$$
\ln \left\langle e^{\lambda A}\right\rangle=\left\langle e^{\lambda A}-1\right\rangle^{c} .
$$

Let us now point out a fundamental relation between cumulants and a quasiclassical theory. It is a characteristic property of quantum mechanics, that the expectation value of a product of two observables $A_{1}$ and $A_{2}$, evaluated with respect to some quantum state $|\psi\rangle$, is generally distinct from a product of the expectation values of $A_{1}$ and $A_{2}$, i.e.,

$$
\left\langle A_{1} A_{2}\right\rangle^{c}=\left\langle A_{1} A_{2}\right\rangle-\left\langle A_{1}\right\rangle\left\langle A_{2}\right\rangle \neq 0,
$$

whereas equality is achieved in the classical limit. Thus one expects that the theory formulated in terms of cumulants will yield a quasiclassical expansion.

To this end, we rewrite the partition function $\mathcal{Z}$ of the system in the basis of spin coherent states $|\mathbf{n}\rangle$, i.e., maximum-spin states pointing in the direction of the unit vector $\mathbf{n}$. In this basis, the cumulants have a simple form, especially if the spin-operator components $S_{z}$ (axis $z$ along $\mathbf{n}$ ) and $S_{ \pm}=S_{x} \pm i S_{y}$ are used. First, a cumulant vanishes if it is of the form $\left\langle\ldots S_{+}\right\rangle^{c}$, $\left\langle\ldots S_{+} S_{-}\right\rangle^{c},\left\langle\ldots S_{z}\right\rangle^{c}$, etc. Non-vanishing cumulants are

$$
\begin{aligned}
& \left\langle S_{+} S_{z}^{n} S_{-}\right\rangle^{c}=2 S(-1)^{n}, \\
& \left\langle S_{+} S_{z}^{n} S_{+} S_{z}^{m} S_{-} S_{z}^{n^{\prime}} S_{-}\right\rangle^{c}=-4 S(-1)^{n+n^{\prime}}(-2)^{m}, \\
& \left\langle S_{+} S_{+} S_{+} S_{-} S_{-} S_{-}\right\rangle^{c}=3\left\langle S_{+} S_{+} S_{-} S_{+} S_{-} S_{-}\right\rangle^{c}=24 S,
\end{aligned}
$$

etc. The above results can be obtained recurrently, using eq. (11) and commutation relations $\left[S_{z}, S_{-}\right]=-S_{-}$and $\left[S_{+}, S_{-}\right]=2 S_{z}$, which remain valid inside cumulants. For scaled spins $\mathbf{S} / S$, each nonvanishing cumulant containing $n$ spin operators scales as $1 / S^{n-1}$.

In the overcomplete basis of spin coherent states, the unit operator is resolved as

$$
\mathbf{1}=\frac{2 S+1}{4 \pi} \int \mathrm{d} \mathbf{n}|\mathbf{n}\rangle\langle\mathbf{n}| .
$$

Thus, for a single-spin system, the trace of an operator $\hat{A}$ over any complete orthonormal basis $|m\rangle$ may be written in terms of spin coherent states as follows 4

$$
\operatorname{tr} \hat{A}=\sum_{m}\langle m|\hat{A}| m\rangle=\frac{2 S+1}{4 \pi} \int \mathrm{d} \mathbf{n} \sum_{m}\langle m|\hat{A}| \mathbf{n}\rangle\langle\mathbf{n} \mid m\rangle=\frac{2 S+1}{4 \pi} \int \mathrm{d} \mathbf{n}\langle\mathbf{n}|\hat{A}| \mathbf{n}\rangle .
$$

The partition function for a Hamiltonian $\hat{H}$ becomes

$$
\mathcal{Z}=\operatorname{tr} \exp (-\beta \hat{H})=\frac{2 S+1}{4 \pi} \int \mathrm{d} \mathbf{n}\langle\mathbf{n}|\exp (-\beta \hat{H})| \mathbf{n}\rangle, \quad \beta \equiv \frac{1}{T} .
$$

The matrix element inside the integral can be written with the help of eq. (3) as

$$
\langle\mathbf{n}|\exp (-\beta \hat{H})| \mathbf{n}\rangle=\exp [-\beta \mathcal{H}(\mathbf{n}, \beta)], \quad \beta \mathcal{H}(\mathbf{n}, \beta)=\langle\mathbf{n}|1-\exp (-\beta \hat{H})| \mathbf{n}\rangle^{c},
$$


where $\mathcal{H}(\mathbf{n}, \beta)$ can be considered as an effective quasiclassical Hamiltonian. In order to find an expansion of $\mathcal{Z}$ in powers of $1 / S$, we expand the expression for $\mathcal{H}$ in a Taylor series:

$$
\mathcal{H}(\mathbf{n}, \beta)=\langle\mathbf{n}|\hat{H}| \mathbf{n}\rangle^{c}-\frac{\beta}{2 !}\langle\mathbf{n}|\hat{H} \hat{H}| \mathbf{n}\rangle^{c}+\frac{\beta^{2}}{3 !}\langle\mathbf{n}|\hat{H} \hat{H} \hat{H}| \mathbf{n}\rangle^{c}+\ldots
$$

Here the first term on the right-hand side is the classical energy of the spin. As seen from eq. (5), increasing powers of $1 / S$ appear in each order of this expansion. If the quantum corrections in eq. (10) are small, then in most cases one can further $\operatorname{expand} \exp (-\beta \mathcal{H})$ in powers of $1 / S$. In this case the statistical weights of different spin orientations are described by a purely classical Hamiltonian, whereas quantum effects manifest themselves in corrections to the density of states.

As an example, let us consider the problem of a spin in a magnetic field.

$$
\hat{H}=-\mathbf{S} \cdot \mathbf{H} \text {. }
$$

For this model, the effective Hamiltonian $\mathcal{H}(\mathbf{n}, \beta)$ can be computed analytically from eq. (9), if one expresses the coherent states in the basis of states with definite projections of $\mathbf{S}$ on $\mathbf{H}$. This yields

$$
\mathcal{H}=-T \ln \sum_{m=-S}^{S} e^{m \beta H} \frac{(2 S) !}{(S+m) !(S-m) !}\left(\frac{1+\cos \theta}{2}\right)^{S+m}\left(\frac{1-\cos \theta}{2}\right)^{S-m},
$$

where $\theta$ is the polar angle. To obtain the cumulant expansion of $\mathcal{H}$, it is convenient to represent the spin operator $\mathbf{S}$ in eq. (10) in the coordinate system with the $z$ axis along the vector $\mathbf{n}$

$$
\mathbf{S}=\mathbf{n} S_{z}+\mathbf{n}_{+} S_{+}+\mathbf{n}_{-} S_{-}, \quad \mathbf{n}_{ \pm} \equiv\left(\mathbf{n}_{x} \mp i \mathbf{n}_{y}\right) / 2,
$$

where $\mathbf{n}_{x}$ and $\mathbf{n}_{y}$ are appropriate transverse basis vectors. Then cumulant averages of different spin components are done with the use of eq. (5). To order $1 / S^{2}$ one obtains

$$
\mathcal{H}=-\mathbf{n} \cdot \mathbf{h}-\frac{\beta}{4 S}[\mathbf{n} \times \mathbf{h}]^{2}+\frac{\beta^{2}}{12 S^{2}}(\mathbf{n} \cdot \mathbf{h})[\mathbf{n} \times \mathbf{h}]^{2}+O\left(\frac{\beta^{3}}{S^{3}}\right),
$$

where $\mathbf{h} \equiv S \mathbf{H}$ is the scaled magnetic field. A similar quasiclassical Hamiltonian for a particle in a potential $V(x)$ has been recently obtained with the help of cumulants in ref. [3].

The function $\mathcal{Z}$ can be evaluated from eq. (8) as for a classical system by doing the integral over the directions of the vector $\mathbf{n}$. Physical quantities such as the energy $U$ and magnetization $\mathbf{m}$ can be obtained by differentiating $\mathcal{Z}$ with respect to the appropriate parameter. Direct evaluation of observables as classical averages $\langle\ldots\rangle$ must be done with care. For the energy one finds

$$
U=-\frac{\partial \ln \mathcal{Z}}{\partial \beta}=\left\langle\mathcal{H}^{*}\right\rangle, \quad \mathcal{H}^{*}=\frac{\partial(\beta \mathcal{H})}{\partial \beta}=\mathcal{H}-\frac{\beta}{4 S}[\mathbf{n} \times \mathbf{h}]^{2}+O\left(\frac{\beta^{2}}{S^{2}}\right),
$$

whereas the scaled magnetization $\mathbf{m} \equiv\langle\mathbf{S}\rangle / S$ is given by

$$
\mathbf{m}=-\frac{\partial \ln \mathcal{Z}}{\partial(\beta \mathbf{h})}=\left\langle\mathbf{n}^{*}\right\rangle, \quad \mathbf{n}^{*}=-\frac{\partial \mathcal{H}}{\partial \mathbf{h}}=\mathbf{n}-\frac{\beta}{2 S}[\mathbf{n} \times[\mathbf{n} \times \mathbf{h}]]+O\left(\frac{\beta^{2}}{S^{2}}\right) .
$$

One notices that not only the effective quasiclassical Hamiltonian $\mathcal{H}$ but also the averaged quantities contain quantum corrections.

Next we have to find the limits of applicability of the quasiclassical expansion. It is clear that the method will break down at low temperatures. At such temperatures the spin fluctuates by 

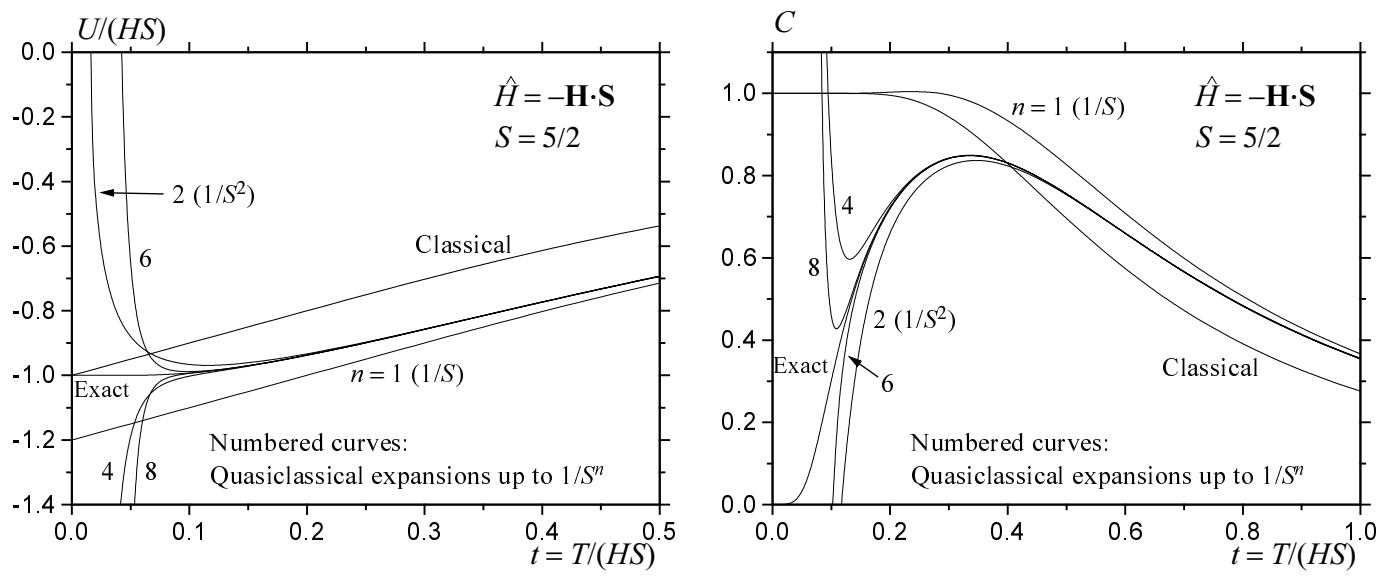

Fig. 1. - Quasiclassical expansions of energy and heat capacity for the spin-in-a-field model, eq. (11).

a small angle $\theta$ from the direction of the field, such that the deviation of the zeroth-order term of $\beta \mathcal{H}$ [see eq. (14)] from the ground state is $\beta h \theta^{2} \sim 1$. The second- and third-order terms of $\beta \mathcal{H}$ become $\beta h \theta^{2}(\beta h / S)$ and $\beta h \theta^{2}(\beta h / S)^{2}$, respectively. Thus the quasiclassical expansion converges for $\beta h / S \lesssim 1$, i.e., for temperatures $T \gtrsim h / S$ which may be well below the classical energy scale $h$ for large spins. For comparison, a usual high-temperature series expansion converges for $T \gtrsim h$. The quantum energy scale $h / S=H$ is defined by the separation of energy levels of the spin in the field. Replacement of discrete energy levels by a continuum of classical spin orientations leads to a temperature dependence of the effective Hamiltonian $\mathcal{H}$ or of the density of states, if $\exp (-\beta \mathcal{H})$ in eq. (8) is expanded in powers of $1 / S$. This expansion is justified under the same condition $\beta h / S \lesssim 1$. For $\beta h / S \gg 1$, i.e., $T \ll h / S$, the spin remains in its ground state, and a continuous quasiclassical expansion becomes invalid. The form of $\mathcal{H}$ in this region is obtained by setting $m=S$ in eq. (12): $\mathcal{H}=-S H-2 S T \ln [(1+\cos \theta) / 2]$.

If $\exp (-\beta \mathcal{H})$ is expanded in powers of $1 / S$, the breakdown of the quasiclassical expansion occurs at lower temperatures and the overall results are better. In this case an analytical calculation is possible. Using eq. (14), one obtains to order $1 / S^{2}$

$$
\ln \mathcal{Z} \cong \ln (2 S)+\ln \left(\frac{\sinh \xi}{\xi}\right)+\frac{\xi B+1}{2 S}+\frac{2 \xi^{2}-3 \xi^{2} B^{2}-6 \xi B-3}{24 S^{2}}+O\left(\frac{1}{S^{3}}\right)
$$

where $\xi \equiv \beta h$ and $B=B(\xi)=\operatorname{coth} \xi-1 / \xi$ is the Langevin function. Quasiclassical expansions of different order in $1 / S$ for the energy and heat capacity of the spin-in-a-field model are shown in fig. 1. Expansions of orders higher than two have been obtained directly by expanding the exact partition function $\mathcal{Z}=\sinh \{\xi[1+1 /(2 S)]\} / \sinh [\xi /(2 S)]$ in powers of $1 / S$. It is seen that already the second quantum approximation nearly quantitatively reproduces the maximum of the heat capacity, although the spin value $S=5 / 2$ is not very high.

As a second example, let us consider the model of two ferro- or antiferromagnetically coupled spins

$$
\hat{H}=\mp J \mathbf{S}_{1} \cdot \mathbf{S}_{2} \text {. }
$$

This model is exactly solvable. The eigenstates labelled by the total spin $n=0,1, \ldots, 2 S$ have the energy $E_{n}=\mp J[n(n+1) / 2-S(S+1)]$ and degeneracy $2 n+1$. The ferro- and 

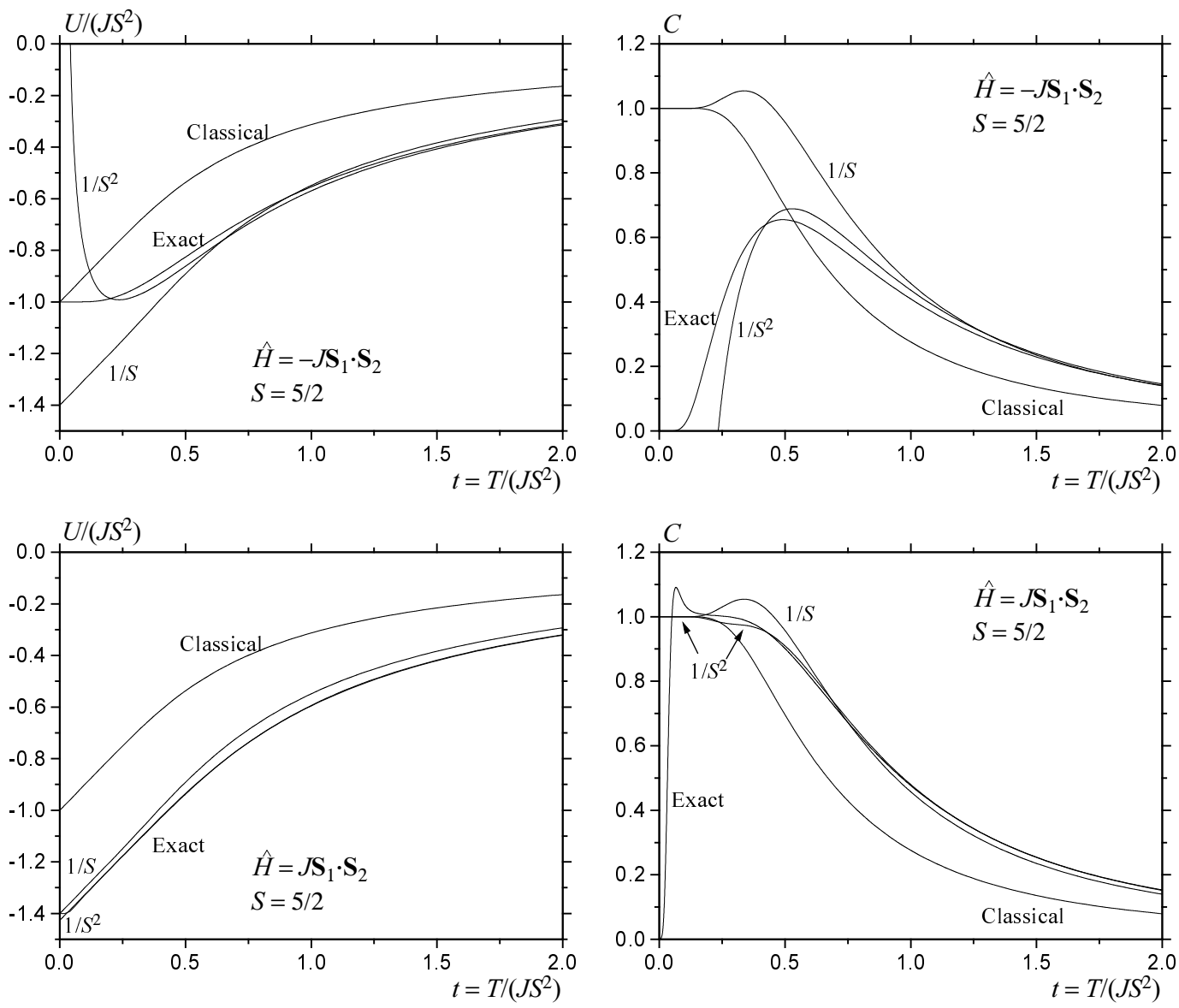

Fig. 2. - Quasiclassical expansion for the energy and heat capacity of the ferro- and antiferromagnetic two-spin models, eq. (18). For the antiferromagnetic coupling, the $1 / S$ approximation reproduces the exact ground-state energy, $U_{0}=-1.4 \tilde{J}$, whereas the $1 / S^{2}$ approximation gives $U_{0}=-1.442666 \tilde{J}$.

antiferromagnetic ground states are $E_{2 S}=-\tilde{J}$ and $E_{0}=-\tilde{J}(1+1 / S)$, respectively, where $\tilde{J} \equiv J S^{2}$ is the classical energy scale. The separation of levels near the ground state is $\Delta E \sim J S=\tilde{J} / S$ for the ferro- and $\Delta E \sim J=\tilde{J} / S^{2}$ for the antiferromagnet.

Derivation of the effective Hamiltonian $\mathcal{H}\left(\mathbf{n}_{1}, \mathbf{n}_{2}\right)$ for this model, where $\mathbf{n}_{1,2}$ are the coherent-state vectors at both sites, proceeds along the same lines. Eqs. (8)-(10) retain their form, only the matrix elements are defined with respect to both coherent states $\mathbf{n}_{1}$ and $\mathbf{n}_{2}$. The latter slightly modifies the routine of calculating the cumulants of the Hamiltonian $\hat{H}$ : In eq. (10) cumulants of $\hat{H}$ are rewritten explicitly according to eq. (11), then independent averages on sites 1 and 2 are taken. After that spins $\mathbf{S}_{1}$ and $\mathbf{S}_{2}$ are expressed in their own coherent-state coordinate systems and the matrix elements of their components $S_{1 \alpha_{1}}$ and $S_{2 \alpha_{2}}$ are computed with the help of cumulants. In a cumulant expansion of eq. (10), this modification results in the appearance of terms carrying higher powers of $1 / S$, in addition to the leading terms of 
order $(\beta / S)^{n}$. To order $1 / S^{2}$ one obtains

$$
\begin{aligned}
\mathcal{H}= & \mp \tilde{J} \mathbf{n}_{1} \cdot \mathbf{n}_{2}-\frac{\beta \tilde{J}^{2}}{2 S}\left[1-\left(\mathbf{n}_{1} \cdot \mathbf{n}_{2}\right)^{2}\right]-\frac{\beta \tilde{J}^{2}}{8 S^{2}}\left[1-\mathbf{n}_{1} \cdot \mathbf{n}_{2}\right]^{2} \\
& \mp \frac{\beta^{2} \tilde{J}^{3}}{12 S^{2}}\left[1-\left(\mathbf{n}_{1} \cdot \mathbf{n}_{2}\right)^{2}\right]\left[1-5 \mathbf{n}_{1} \cdot \mathbf{n}_{2}\right]+O\left(\frac{1}{S^{3}}\right) .
\end{aligned}
$$

Note that quantum corrections have a non-Heisenberg form. As follows from the cumulant expansion of eq. (10), an additional pair $\mathbf{n}_{1} \cdot \mathbf{n}_{2}$ is associated with each further power of $\beta$. Eq. (19) results in the expansion for $\ln \mathcal{Z}$ of the form

$$
\ln \mathcal{Z} \cong 2 \ln (2 S)+\ln \left(\frac{\sinh \xi}{\xi}\right)+\frac{\xi B+1}{S}+\frac{5 \xi^{2}-6 \xi^{2} B^{2} \mp \xi^{2} B-9 \xi B-3}{12 S^{2}}+O\left(\frac{1}{S^{3}}\right),
$$

where $\xi \equiv \beta \tilde{J}$.

Quasiclassical expansions for the energy and heat capacity of the two-spin model are shown in fig. 2. They are obtained by differentiating eq. (20). For ferromagnetic coupling the results resemble those for the spin-in-a-field model, although here quantum corrections are about two times larger. For that model, the leading asymptote $U \cong-h \xi(1+1 / S) / 3$ at high temperatures is recovered already at order $1 / S$, whereas the proper leading asymptote $U \cong-\tilde{J} \xi(1+1 / S)^{2} / 3$ for the two-spin model is obtained only at order $1 / S^{2}$.

For antiferromagnetic coupling and to order $1 / S^{2}, U$ and $C=\mathrm{d} U / \mathrm{d} T$ do not diverge as $T \rightarrow 0$, because the leading $\xi^{2}$ terms in eq. (20) cancel. The accuracy of the $1 / S^{2}$ approximation over a wide range of temperatures is quite impressive. It is tempting to explain this by the fact that for antiferromagnetic coupling the separation of the low-lying energy levels is by a factor of $1 / S \ll 1$ smaller than for ferromagnetic coupling, as was mentioned above. Therefore the properties of the system are expected to be "more classical".

Furthermore, one could speculate that for many-spin systems without an energy gap the quasiclassical expansion works even better. However, it is not true. The quasiclassical expansion without a resummation does not reproduce rational powers of $T$, which appear, e.g., in linear spin-wave theory. Therefore, it should break down below the quantum energy scale $J S=\tilde{J} / S$. Note that the SWT based either on the Holstein-Primakoff or path-integral formalisms (see, e.g., ref. [1]) works in the range $T \lesssim \tilde{J}$. Its results should overlap with those of the cumulant expansion in the range $\tilde{J} / S \lesssim T \lesssim \tilde{J}$. It is interesting to speculate how $\beta / S$ corrections of the cumulant expansion go over to $1 / S$ corrections of the SWT (e.g., for antiferromagnets) when $T \lesssim \tilde{J} / S$. One would expect that the leading terms $(\beta / S)^{n}$ of eq. (19) convert to the leading order of the SWT, whereas $\beta / S^{2}$ and other subdominant terms result in $1 / S$ spin-wave corrections.

We have shown that for spin systems with large $S$ an application of the cumulant technique together with spin coherent states leads to effective, classical-like Hamiltonians with quantum corrections taken into account. These Hamiltonians can be further treated by a number of methods for classical spin systems, such as exact solutions, Monte-Carlo simulations, or the classical-spin diagram technique [5], which is again based on cumulants.

\section{REFERENCES}

[1] Auerbach A., Interacting Electrons and Quantum Magnetism, (Springer, Berlin) 1994.

[2] Fulde P. , Electron Correlations in Molecules and Solids, (Springer, Berlin) 1995.

[3] Kladko K. And Fulde P. , Int. Journ. Quant. Chem., 66 (1998) 377; cond-mat/9709044 Eprint, 1997. 
[4] Lieb E. H., Comm. Math. Phys., 31 (1973) 559.

[5] Garanin D. A., Phys. Rev. B, 53 (1996) 11593. 